\section{Outcomes following cataract surgery in choroideremia}

${ }^{1}$ Oxford Eye Hospital and Nuffield Laboratory of Ophthalmology, John Radcliffe Hospital, Oxford, UK

${ }^{2}$ Moorfields Eye Hospital Foundation Trust, NIHR Ophthalmology Biomedical Research Centre, London, UK

Correspondence: TL Edwards, Nuffield Department of Clinical Neurosciences, Level 6, West Wing, John Radcliffe Hospital, Headley Way, Oxford OX39DU, UK Tel: +44 (0)18 65231513 E-mail: thomas.edwards@ cantab.net

Received: 24 May 2014 Accepted in revised form: 2 November 2014 Published online: 16 January 2015

\begin{abstract}
Purpose To present a case series of cataract surgery outcomes in choroideremia eyes with an emphasis on the safety of this common operation in advanced stages of the disease. Methods A single centre retrospective interventional case series comprising six patients with varying degrees of visual loss secondary to choroideremia underwent cataract surgery at a single tertiary eye hospital. Pre- and post-operative bestcorrected Snellen visual acuity, spectral domain optical coherence tomography (SD-OCT), and slit lamp examination were performed together with fundus autofluorescence (FAF) and colour fundus photographs. The prevalence of intra- or postoperative complications, post-operative visual outcome, and change in central macular thickness were recorded.

Results The pre-operative best-corrected Snellen visual acuity in the operated eyes ranged from 6/12 (20/40) to PL. All but one patient had either an objective or a subjective improvement in visual acuity. There was no evidence of retinal phototoxicity or post-operative cystoid macular oedema (CMO). Three patients developed early capsular fibrosis. Conclusions Although the residual functioning retina in choroideremia patients may be potentially vulnerable, this report finds no evidence of iatrogenic vision loss after uncomplicated cataract surgery. This suggests that cataract surgery may be performed safely in choroideremia patients, although a guarded prognosis for visual improvement should be emphasized in the informed consent.
\end{abstract} Eye (2015) 29, 460-464; doi:10.1038/eye.2014.326; published online 16 January 2015

\section{Introduction}

Choroideremia is an X-linked recessive retinal degeneration that results in bilateral progressive loss of vision with onset typically in the second decade of life. ${ }^{1}$ It is characterized by a degeneration of the retinal pigment epithelium (RPE), followed by the choriocapillaris and outer retina, ${ }^{2}$ which slowly advances towards the posterior pole. Symptoms thus evolve from night blindness in the early stages to concentric field loss that encroaches on the macula over time, with central vision sometimes preserved until the seventh decade. ${ }^{3}$ The disease has an estimated prevalence of $1: 50000{ }^{4}$

Choroideremia is caused by a loss of function mutation in the CHM gene, which encodes the ubiquitously expressed 73.5 kDa RabGTPase escort protein 1 (REP1). RabGTPase (Rab) proteins are crucial for mediating the intracellular destination of transport vesicles ${ }^{5}$ and in part, their trafficking specificity relies on prenylation by Rab geranylgeranyl transferase (RabGGTase), which requires REP1. The onset of severe visual loss from retinal degeneration may coincide with cataract formation, given the natural history of both conditions, prompting a patient enquiry regarding the safety and efficacy of cataract surgery. In addition, should gene therapy for choroideremia ${ }^{6}$ become the future standard of care for selected patients, post-vitrectomy cataract ${ }^{7,8}$ will become more common. It is therefore important to establish whether choroideremia eyes are particularly vulnerable during and after cataract surgery. The small area of residual functioning retina, for example, may be susceptible to microscope light-induced phototoxicity or post-operative CMO. For this reason, we performed a detailed retrospective analysis of cataract surgery performed in choroideremia patients.

\section{Materials and methods}

Six patients with confirmed choroideremia who underwent uncomplicated cataract surgery in a single surgical unit between September 2011 and March 2014 were identified. They were 
recruited from a cohort of 65 patients seen at the Oxford Eye Hospital as part of an ongoing multi-centre UK-based gene therapy trial (www.clinicaltrials.gov, NCT01461213). However, none of these particular six patients had received gene therapy, as no trial patients have yet undergone cataract surgery. IRB approval (where relevant-for example, genetic testing) was covered by NCT01461213 and the study adhered to the tenets of the Declaration of Helsinki. Fundus autofluorescence, SD-OCT, and best-corrected Snellen visual acuity (VA) were recorded for all patients pre-operatively. The latter two tests were repeated post-operatively. Biometry was performed using laser interferometry with an IOL Master A-scan (Carl Zeiss Ltd, Cambridge, UK). Informed consent for standard cataract surgery was obtained from all patients. Exposure to potential retinal phototoxicity from the operating microscope was kept to a minimum throughout each case, as is routine for all patients with retinal degenerations in the study centre. Surgery was performed under topical anaesthesia with isotonic intracameral lignocaine in patients 2,3 , and 5; topical only in patients 4 and 6; and sub-tenons 50:50 mix of bupivicaine and lignocaine at a final concentration of 0.25 and $1 \%$, respectively, with hyaluronidase $1500 \mathrm{IU}$ in patient 1 . An Infiniti phacoemulsification machine (Alcon Laboratories, Fort Worth, TX, USA) was used and post-operative topical steroid and broad-spectrum antibiotic were prescribed to the operated eye for 1 month in all cases. A paired Student's t-test was performed to compare mean central retinal thickness pre- and post-surgery.

\section{Results}

The visual acuities, demographic characteristics, and CHM gene mutations of each patient are shown in Table 1. Five patients were of Caucasian background and a sixth was of Indian subcontinent descent. The median age (SD) was 54 (8) years, (range 49-70 years). All had advanced or end-stage retinal degeneration with no CMO. Cataract in patients 1 and 2 developed after 23 gauge pars plana vitrectomy and inner limiting membrane peel for full-thickness macular hole (FTMH) repair. Neither FTMH was associated with posterior retinal detachment, although this has been reported in choroideremia, 9,10 and both achieved successful anatomical closure. Fundus imaging representative of the cohort is shown for patients 2 and 3 (see Figure 1). A trace amount of pre-operative intraretinal cystic degenerative change was seen by SD-OCT in patient 4 only. Uncomplicated phacoemulsification was performed in all the six patients with placement of an intra-ocular lens (IOL) in the capsular bag. In view of the possibility of patients becoming entirely dependent on intrinsically photosensitive retinal ganglion cells (ipRGC) for their circadian function, blue light lenses were avoided because little is known about the effects of these lenses in patients with no photoreceptor function. Post-operative fundoscopic examination showed no sign of surgeryinduced $\mathrm{CMO}$ in any patient, which was confirmed by SD-OCT. There was no significant change in the median central retinal thickness (CRT), which was $163 \mu \mathrm{m}$ (SD $=79$, range 79-273 $\mu \mathrm{m}$ ) before surgery and $172 \mu \mathrm{m}$ $(\mathrm{SD}=78$, range $75-267 \mu \mathrm{m})$ at 1 month post surgery $(P=0.95)$.

Patient 2 reported a subjective improvement in his VA although it remained at ' $\mathrm{HM}^{\prime}$ post surgery. A thickened posterior capsule was noted intra-operatively and at 6 months, anterior capsularphimosis was evident with mild superior displacement of the IOL. Similarly, patient 1 noted some improvement in subjective acuity although objectively it remained 6/24. He developed early posterior capsular opacification, which required Nd:YAG laser capsulotomy. Patient 3 noted a slight improvement in VA in the operated eye for 1-2 days before a return to pre-operative levels. He described being able to see 'fingers moving', after being only 'light perception' before the operation. Unfortunately this was not sustained. He also described significant photophobia during the first week. Similarly, Patient 4 noticed a transient improvement in his VA for 3 days before it became more varied. It was subjectively better in the mornings. He developed significant early anterior

Table 1 Clinical data and CHM gene mutation

\begin{tabular}{lclcccll}
\hline Patient & Age & CHM mutation & Pre-op VA & Post-op VA & Fellow eye VA & IOL & Past surgery \\
\hline 1 & 54 & c.1079delA & $6 / 24(20 / 80)$ & $6 / 24(20 / 80)$ & $6 / 60(20 / 200)$ & SA60AT & 23G PPV, ILM peel for FTMH \\
2 & 49 & c.1300_1303delGTGG & HM & HM & $6 / 12(20 / 40)$ & Tecnis Z9002 & 23G PPV, ILM peel for FTMH \\
3 & 50 & EX10_11del & PL & PL & $6 / 60(20 / 200)$ & Tecnis Z9002 & Nil \\
4 & 70 & c.757C $>$ T & $6 / 12(20 / 40)$ & $6 / 9(20 / 32)$ & $6 / 9(20 / 32)$ & Tecnis Z9002 & Nil \\
5 & 64 & c.819+1G $>$ T & $6 / 60(20 / 200)$ & $6 / 18(20 / 63)$ & HM & Akreos Adapt & Nil \\
6 & 58 & c.49+3del & HM & $6 / 18(20 / 63)$ & CF & Tecnis Z9002 & Nil \\
\hline
\end{tabular}

FTMH, full-thickness macular hole; 23G PPV, 23 gauge pars plana vitrectomy; ILM, inner limiting membrane; VA, visual acuity . SA60AT (Alcon, Fort Worth Laboratories, TX), Technis Z9002 (Abbott Medical Optics, Santa Ana, CA), Akreos Adapt (Bausch and Lomb, Rochester, NY). 

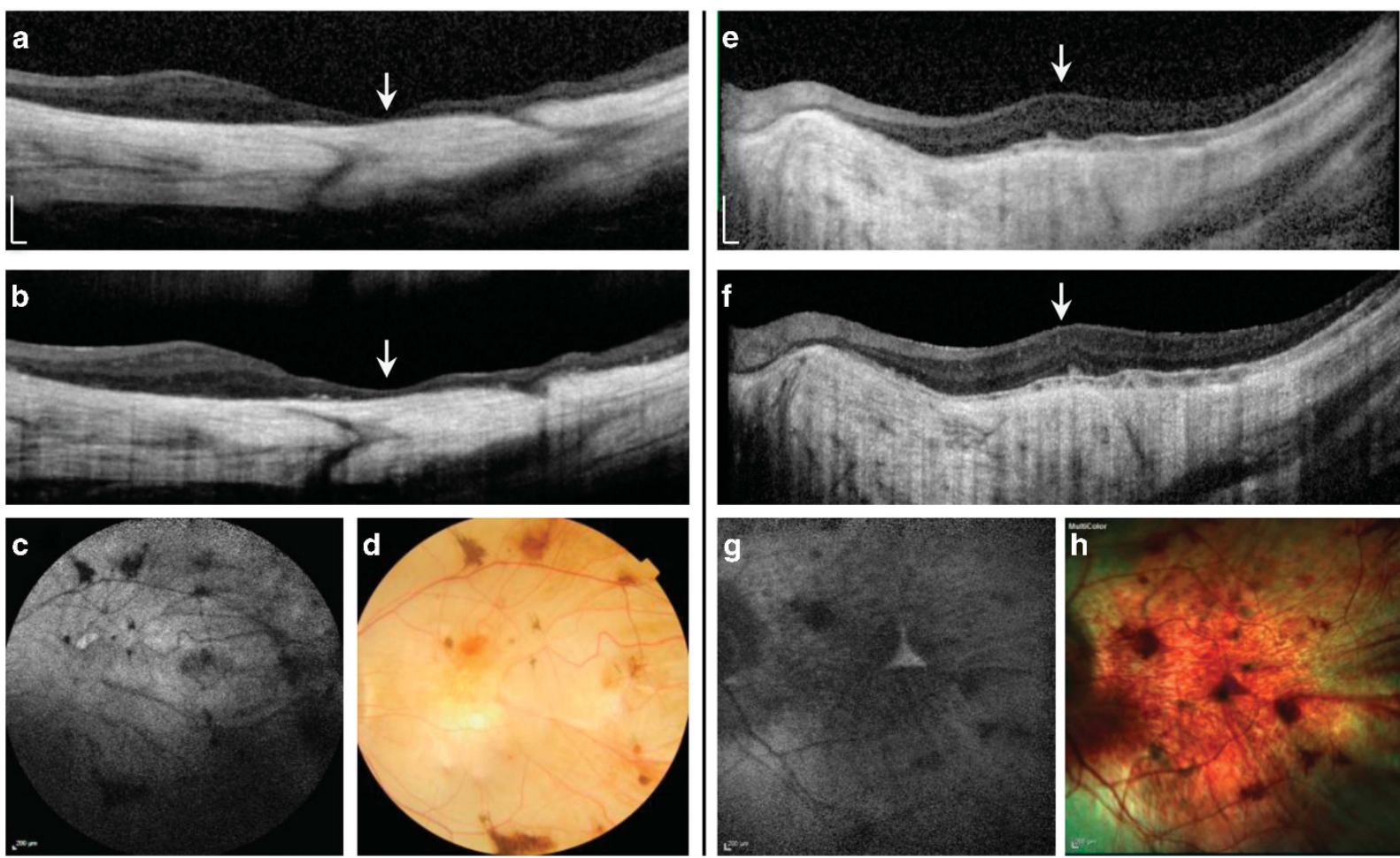

Figure 1 Pre- and post-operative spectral domain optical coherence tomography (SD-OCT), with fundus autofluorescence (FAF), and colour fundus photos of the operated eyes of patient $2(\mathrm{a}-\mathrm{d})$ and $3(\mathrm{e}-\mathrm{h})$. (a) SD-OCT horizontal scan through the macular centre (arrow) showing gross thinning of the choroid and overlying retina with no visible intra- or sub-retinal fluid and no significant change in the post-operative scan (b). (c) $55^{\circ}$ FAF and colour fundus photos showing widespread profound chorioretinal atrophy with attenuation of the retinal vasculature and pigment clumping. (e) Horizontal SD-OCT through the macular centre (arrow) of patient 3 showing a relatively thicker retina but significant choroidal atrophy. No post-operative $\mathrm{CMO}$ or retinal thinning was seen (f). $30^{\circ} \mathrm{FAF}$ and colour fundus photos $(\mathrm{g}-\mathrm{h})$ show the very small central triangular region of remaining retina, which is hyperautofluorescent on FAF.

capsularphimosis with posterior displacement of the IOL, and a large vitreous floater that intermittently obstructed the visual axis. Five months post cataract surgery, he returned to the theatre for anterior capsulotomy and 23 gauge vitrectomy, which improved the VA to 6/9 (20/32). Patient 5 improved from 6/60 $(20 / 200)$ to $6 / 18(20 / 63)$ after surgery, and last, patient 6 had an uneventful course with improvement in his VA to $6 / 18(20 / 63)$ from HM vision after the removal of a posterior sub-capsular cataract.

\section{Discussion}

It is important to document that cataract surgery is safe in eyes with choroideremia given its potential to optimize vision in the late stages of the disease as senile cataract develops. In addition, iatrogenic cataract secondary to vitrectomy may become more frequent in choroideremia patients with the introduction of subretinal gene therapy, which currently requires removal of the vitreous to access the submacular space. To our knowledge this is the first published series of cataract surgery in choroideremia. Potential surgical risk factors in these eyes might include (i) higher rate of postoperative $\mathrm{CMO}$; (ii) retinal phototoxicity from the operating microscope; (iii) weak zonules; or (iv) higher rates of post-operative capsular fibrosis. Although only a small series, this study suggests that cataract surgery in choroideremia carries no specific additional risk of intraor early post-operative complications, although early capsularphimosis may be a feature.

There have been a small number of retrospective studies examining cataract surgery in other heritable retinal degenerations such as retinitis pigmentosa and Usher syndrome, which showed that these patients generally had good outcomes. ${ }^{11,12}$ A retrospective analysis of 142 eyes of 89 patients with retinitis pigmentosa ${ }^{13}$ reported a $14 \%$ rate of post-operative $\mathrm{CMO}$ as detected by fluorescein angiography. This compared with rates detected by SD-OCT in normal eyes after cataract surgery of between 4 and $11 \% .{ }^{14,15}$ Although $\mathrm{CMO}$ is a recognized late complication of choroideremia itself, ${ }^{16}$ no patients in this cohort developed postoperative CMO. 
Two patients developed anterior capsularphimosis (patients 2 and 4) and another (patient 1) required Nd:YAG laser capsulotomy for posterior capsular opacification, although no eyes exhibited significant zonular laxity. By comparison, aggressive capsularphimosis, zonular weakness and IOL dislocation have all been reported in retinitis pigmentosa. ${ }^{13,17-23}$ Phototoxicity is a serious consideration in the context of attenuated photoreceptor and RPE function as seen in choroideremia. The main risk factor for phototoxicity is the duration of surgery, ${ }^{24,25}$ which was kept to a minimum in all the patients and the eye was shielded from the microscope light during the pauses in operating. There was no subjective or objective evidence of retinal phototoxicity in this cohort.

An interesting observation was the transient subjective improvement in VA noted by patients 3 and 4 immediately after their surgery. One might speculate that this signalled an element of reversibility to the chorioretinal degeneration, if only short-lived, that was somehow induced by the cataract surgery. However, the early deterioration seen in patient 4 may have been due to a new vitreous floater obscuring the small central island of functional retina (with later exacerbation by anterior capsularphimosis). Improvement in choroideremia is not without precedent: in a phase 1 trial sustained improvement of 13 letters was noticed in a patient with choroideremia 24 weeks after the implantation of intravitreal encapsulated ciliary neurotrophic factor, ${ }^{26}$ which perhaps boosted visual function in cells that had not yet undergone irreversible degeneration. In this study, the mechanism by which cataract surgery leads to transient improvement was unclear, but a transient surgeryinduced elevation in the intra-ocular growth factors is one possible mechanism. This is possible as some purified lens proteins, for example, $\beta$ - and $\gamma$-crystallin have been shown to stimulate the release of neuroprotective factors such as ciliary neurotropic factor (CNTF) in animal models. ${ }^{27}$

In conclusion, this retrospective surgical case series has shown that for routine cataract surgery, potential choroideremia-specific sight-threatening complications such as retinal phototoxicity or CMO did not eventuate, although capsule-related changes were noted. None of the patients lost vision as a result of cataract surgery and all but one patient had an objective or subjective improvement in visual acuity, although maintenance of these gains may be limited by progression of retinal degeneration. We would recommend proceeding with cataract surgery in patients with choroideremia and visually significant cataract after discussing the guarded prognosis for improvement in VA as part of the consent process.

\section{Summary}

\section{What was known before}

- Safety of cataract surgery in choroideremia eyes has not been previously published.

- Important to document whether choroideremia eyes are vulnerable to particular specific complications.

What this study adds

- No specific intra- or post-operative complications to which choroideremia eyes might be predisposed were observed in this series, for example, light microscopeinduced retinal phototoxicity or post-operative cystoid macular oedema.

- However, three eyes developed early capsular fibrosis.

- The visual outcome was limited by the extent of chorioretinal degeneration.

- On the basis of these data, cataract surgery with a guarded prognosis for visual improvement is recommended in choroideremia eyes with visually significant cataract.

\section{Conflict of interest}

REM is a Director of NightstarX Ltd (215 Euston Road, London, UK NW1 2BE), a choroideremia gene therapy company established and funded by the Wellcome Trust. The remaining authors declare no conflict of interest.

\section{References}

1 Rosenberg T, Schwartz M. Age differences of visual field impairment and mutation spectrum in Danish choroideremia patients. Acta Ophthalmol (Copenh) 1994; 72(6): 678-682.

2 MacDonald IM, Russell L, Chan C-C. Choroideremia: new findings from ocular pathology and review of recent literature. Surv Ophthalmol 2009; 54(3): 401-407.

3 Roberts MF, Fishman GA, Roberts DK, Heckenlively JR, Weleber RG, Anderson RJ et al. Retrospective, longitudinal, and cross sectional study of visual acuity impairment in choroideraemia. Br J Ophthalmol 2002; 86(6): 658-662.

4 Pagon RA, Adam MP, Ardinger HH, Bird TD, Dolan CR, Fong CT et al. Choroideremia. University of Washington, Seattle: Seattle, WA, USA, 1993.

5 Cai H, Reinisch K, Ferro-Novick S. Coats, tethers, Rabs, and SNAREs work together to mediate the intracellular destination of a transport vesicle. Dev Cell 2007; 12(5): 671-682.

6 MacLaren RE, Groppe M, Barnard AR, Cottriall CL, Tolmachova T, Seymour L et al. Retinal gene therapy in patients with choroideremia: initial findings from a phase 1/2 clinical trial. Lancet 2014; 383(9923): 1129-1137.

7 Hsuan JD, Brown NA, Bron AJ, Patel CK, Rosen PH. Posterior subcapsular and nuclear cataract after vitrectomy. J Cataract Refract Surg 2001; 27(3): 437-444.

8 Cherfan GM, Michels RG, de Bustros S, Enger C, Glaser BM. Nuclear sclerotic cataract after vitrectomy for idiopathic epiretinal membranes causing macular pucker. Am J Ophthalmol 1991; 111(4): 434-438.

9 Shinoda H, Koto T, Fujiki K, Murakami A, Tsubota K, Ozawa Y. Clinical findings in a choroideremia patient who 
underwent vitrectomy for retinal detachment associated with macular hole. Jpn J Ophthalmol 2011; 55(2): 169-171.

10 Zinkernagel MS, Groppe M, MacLaren RE. Macular hole surgery in patients with end-stage choroideremia. Ophthalmology 2013; 120(8): 1592-1596.

11 Fagerholm PP, Philipson BT. Cataract in retinitis pigmentosa An analysis of cataract surgery results and pathological lens changes. Acta Ophthalmol (Copenh) 1985; 63(1): 50-58.

12 Newsome DA, Stark WJ, Maumenee IH. Cataract extraction and intraocular lens implantation in patients with retinitis pigmentosa or Usher's syndrome. Arch Ophthalmol 1986; 104(6): 852-854.

13 Jackson H, Garway-Heath D, Rosen P, Bird AC, Tuft SJ. Outcome of cataract surgery in patients with retinitis pigmentosa. Br J Ophthalmol 2001; 85(8): 936-938.

14 Bélair M-L, Kim SJ, Thorne JE, Dunn JP, Kedhar SR, Brown DM et al. Incidence of cystoid macular edema after cataract surgery in patients with and without uveitis using optical coherence tomography. Am J Ophthalmol 2009; 148(1): 128-135.e2.

15 Perente I, Utine CA, Ozturker C, Cakir M, Kaya V, Eren H et al. Evaluation of macular changes after uncomplicated phacoemulsification surgery by optical coherence tomography. Curr Eye Res 2007; 32(3): 241-247.

16 Genead MA, Fishman GA. Cystic macular oedema on spectral-domain optical coherence tomography in choroideremia patients without cystic changes on fundus examination. Eye 2011; 25(1): 84-90.

17 Lee HJ, Min S-H, Kim TY. Bilateral spontaneous dislocation of intraocular lenses within the capsular bag in a retinitis pigmentosa patient. Korean J Ophthalmol 2004; 18(1): 52-57.

18 Tomasz Z, Anna L-I. Bilateral spontaneous dislocation of IOLs within the capsular bag to the anterior chamber in a retinitis pigmentosa patient. Klin Oczna 2011; 113(10-12): 373-375.
19 Nikpoor N, Stone DU. Rapid capsular phimosis in retinitis pigmentosa. Ophthalmic Surg Lasers Imaging 2010; 41 Online.

20 Dikopf MS, Chow CC, Mieler WF, Tu EY. Cataract extraction outcomes and the prevalence of zonular insufficiency in retinitis pigmentosa. Am J Ophthalmol 2013; 156(1): 82-88.

21 Jin-Poi T, Shatriah I, Khairy-Shamel ST, Zunaina E. Rapid anterior capsular contraction after phacoemulsification surgery in a patient with retinitis pigmentosa. Clin Ophthalmol 2013; 7: 839-842.

22 Bayyoud T, Bartz-Schmidt KU, Yoeruek E. Long-term clinical results after cataract surgery with and without capsular tension ring in patients with retinitis pigmentosa: a retrospective study. BMJ Open 2013; 3(4): e002616.

23 Hayashi K, Hayashi H, Matsuo K, Nakao F, Hayashi F. Anterior capsule contraction and intraocular lens dislocation after implant surgery in eyes with retinitis pigmentosa. Ophthalmology 1998; 105(7): 1239-1243.

24 Khwarg SG, Linstone FA, Daniels SA, Isenberg SJ, Hanscom TA, Geoghegan M et al. Incidence, risk factors, and morphology in operating microscope light retinopathy. Am J Ophthalmol 1987; 103(3 Pt 1): 255-263.

25 Lee BL, Sternberg P. Microscope light toxicity. Semin Ophthalmol 1993; 8(2): 151-155.

26 Sieving PA, Caruso RC, Tao W, Coleman HR, Thompson DJ, Fullmer KR et al. Ciliary neurotrophic factor (CNTF) for human retinal degeneration: phase I trial of CNTF delivered by encapsulated cell intraocular implants. Proc Natl Acad Sci USA 2006; 103(10): 3896-3901.

27 Fischer D, Hauk TG, Müller A, Thanos S. Crystallins of the beta/gamma-superfamily mimic the effects of lens injury and promote axon regeneration. Mol Cell Neurosci 2008; 37(3): 471-479. 